\title{
A TRANSITIVE MEDIAL SUBSPACE LATTICE
}

\section{K. J. HARRISON, HEYDAR RADJAVI AND PETER ROSENTHAL}

ABSTRACr. We give an example of a transitive medial lattice of subspaces of Hilbert space which has four nontrivial elements.

Let $\mathfrak{F C}$ be a separable infinite-dimensional Hilbert space (real or complex). A subspace of $\mathfrak{H C}$ is a closed linear manifold. If $A$ is a bounded linear operator on $\mathfrak{H C}$, then the collection of all subspaces invariant under $A$ is a lattice. There has been some study of the lattices that can occur as invariant subspace lattices (cf. [2] and references given there).

Recently Halmos [1] initiated the study of the lattices that can occur as the invariant subspace lattices of collections of operators.

A subspace lattice is transitive if the only bounded operators which leave invariant all the subspaces in the lattice are the multiples of the identity operator. The basic problem of characterizing transitive lattices seems to be difficult. A lattice with 0 and 1 is said to be medial if every pair of distinct nontrivial (i.e. neither 0 nor 1) elements are complements of each other. Halmos [1] has given an example of a transitive medial subspace lattice with 5 nontrivial elements, and has shown that every transitive medial lattice of subspaces of a finite-dimensional space of (complex) dimension greater than 2 has at least 5 nontrivial elements.

In this note we give an example of a transitive medial lattice of subspaces of separable Hilbert space which has 4 nontrivial elements. We think that the number 4 is minimal, but we have been unable to prove it.

To construct the example let $\Re$ be a Hilbert space with orthonormal basis $\left\{e_{n}\right\}_{n=-\infty}^{\infty}$. Let $\mathfrak{K}=\mathscr{K} \oplus \mathcal{K}$ and let $\mathfrak{T}_{x 0}, \mathfrak{T}_{0 x}$ and $\mathfrak{T}_{x x}$ denote the subspaces $\mathscr{K} \oplus 0,0 \oplus \mathcal{K}$ and $\{(x, x): x \in \mathcal{K}\}$ of $\mathcal{K}$. Let $\alpha_{n}=1$ for $n \leqq 0$ and $\alpha_{n}=\exp \left((-1)^{n} n !\right)$ for $n>0$. Let $T$ be the operator defined by

$$
T\left(\sum_{n=-\infty}^{\infty} y_{n} e_{n}\right)=\left(\sum_{n=-\infty}^{\infty} y_{n} \alpha_{n} e_{n+1}\right)
$$

on the domain $D=\left\{\sum_{n=-\infty}^{\infty} y_{n} e_{n}: \sum_{n=-\infty}^{\infty}\left|y_{n} \alpha_{n}\right|^{2}<\infty\right\}$. Then it is

Received by the editors September 8, 1970.

AMS 1969 subject classifications. Primary 4735; Secondary 4665, 0630.

Key words and phrases. Hilbert space, operator, invariant subspace, lattice, transitive. 
easily verified that $T$ is a closed operator, i.e., that the subspace $\mathfrak{N}_{T}=\{(x, T x) ; x \in \mathfrak{D}\}$ is a closed subspace of $\mathfrak{H}$.

THEOREM. The set $\left\{\{0\}, \mathcal{H}, \mathfrak{N}_{x 0}, \mathfrak{N}_{0 x}, \mathfrak{N}_{x x}, \mathfrak{N}_{T}\right\}$ is a transitive medial lattice.

Proof. We first show that the set is a medial lattice. For this we shall need some facts about $T$ :

(i) The closure of the range of $T$ is $\mathfrak{K}$, since $e_{n} \in$ range $T$ for all $n$.

(ii) The closure of the range of $(T-1)$ is $\mathcal{K}$, since if $x=\sum x_{n} e_{n}$ is orthogonal to $(T-1) e_{n}$ for all $n$, then $\alpha_{n} x_{n+1}-x_{n}=0$ for all $n$. Therefore, since $\alpha_{n}=1$ for $n \leqq 0, x_{n}=x_{0}$ for all negative $n$ and thus $x_{0}=0$. Since every $\alpha_{n}$ is positive, it follows that $x_{n}=0$ for all $n$.

(iii) Null $T=\{0\}$, since $\alpha_{n} \neq 0$ for all $n$.

(iv) Null $(T-1)=\{0\}$, for if $(T-1) \sum x_{n} e_{n}=0$, then $x_{n+1}=\alpha_{n} x_{n}$, and, as in (ii), we get $x_{n}=0$ for all $n$.

We must show that any two nontrivial elements of the lattice are complementary.

(a) $\mathfrak{M}_{x_{0}} \cap \mathfrak{M T}_{T}=\{0\}$ by (iii), and $\mathfrak{T}_{x 0} \vee \mathfrak{T}_{T}=\mathfrak{H C}$ by (i).

(b) $\mathfrak{M}_{x x} \cap \mathfrak{M}_{T}=\{0\}$ by (iv), and $\mathfrak{M}_{x x} \vee \mathfrak{N T}_{T}=\mathfrak{H C}$ by (ii).

The other pairs are trivially seen to be complementary, and thus the set is a medial lattice.

We now show that the lattice is transitive. Obviously every operator which leaves $\mathfrak{T}_{x 0}, \mathfrak{T}_{0 x}$ and $\mathfrak{N}_{x x}$ invariant can be written $A \oplus A$ where $A$ is an operator on $\Re$. We must show that $A$ is a multiple of the identity. It follows from the invariance of $\mathfrak{T}_{T}$ under $A \oplus A$ that $D$ is invariant under $A$ and that $A T=T A$ on $D$.

Note that for every $x \in D,\left(T x, e_{n}\right)=\alpha_{n-1}\left(x, e_{n-1}\right)$. Since $\left(A T e_{m-1}, e_{n}\right)$ $=\left(T A e_{m-1}, e_{n}\right)$ for all $m$ and $n$, it follows that $\alpha_{m-1}\left(A e_{m}, e_{n}\right)$ $=\alpha_{n-1}\left(A e_{m-1}, e_{n-1}\right)$. Thus $\left(A e_{n}, e_{n}\right)$ is a constant independent of $n$. If $A$ were not a multiple of the identity, there would exist distinct integers $m$ and $n$ such that $\left(A e_{m}, e_{n}\right) \neq 0$. But the above computation implies that

$$
\left(A e_{m+k}, e_{n+k}\right)=\frac{\alpha_{n} \alpha_{n+1} \cdots \alpha_{n+k-1}}{\alpha_{m} \alpha_{m+1} \cdots \alpha_{m+k-1}}\left(A e_{m}, e_{n}\right) \quad \text { for } k>0
$$

The proof will be completed if we show that $\left(A e_{m+k}, e_{n+k}\right)$ is not bounded as $k \rightarrow \infty$, because this would contradict the fact that $A$ is bounded.

The fraction on the right-hand side of the above equation can be rewritten as

$$
c_{1} \alpha_{m+k} \alpha_{m+k-1} \cdots \alpha_{n+k-1} \text { or } c_{2}\left(\alpha_{n+k} \alpha_{n+k+1} \cdots \alpha_{m+k-1}\right)^{-1}
$$


according as $n>m$ or $m>n$, where $c_{1}$ and $c_{2}$ are nonzero numbers not depending on $k$. In both cases the fraction is unbounded: just observe that if $r$ is a fixed integer and if

$$
P_{j}=\alpha_{j} \alpha_{j+1} \cdots \alpha_{j+r}
$$

then $\lim \sup _{j \rightarrow \infty} P_{j}=\infty$ and $\lim \inf _{j \rightarrow \infty} P_{j}=0$.

\section{REFERENCES}

1. Paul R. Halmös, Ten problems in Hilbert space, Bull. Amer. Math. Soc. 76 (1970), 887-933.

2. Peter Rosenthal, Examples of invariant subspace lattices, Duke Math. J. 37 (1970), 103-112. MR 40 \#497.

Monash University, Clayton, Victoria 3168, Australia

University of Toronto, Toronto, Ontario, Canada 